\title{
Transperitoneal versus retroperitoneal laparoscopic adrenalectomy for adrenal tumours in adults (Protocol)
}

\author{
Arezzo A, Cochetti GG, Cirocchi R, Randolph JJ, Mearini EE, Passera R
}
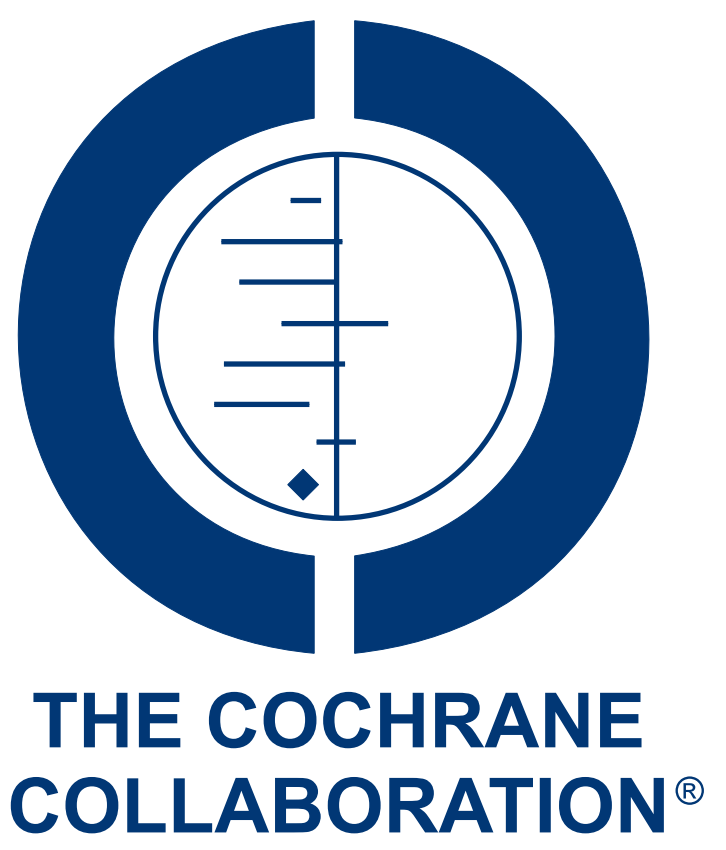

This is a reprint of a Cochrane protocol, prepared and maintained by The Cochrane Collaboration and published in The Cochrane Library 2015, Issue 4

http://www.thecochranelibrary.com

\section{WILEY}

Transperitoneal versus retroperitoneal laparoscopic adrenalectomy for adrenal tumours in adults (Protocol)

Copyright () 2015 The Cochrane Collaboration. Published by John Wiley \& Sons, Ltd. 
TABLE OF CONTENTS

HEADER . . . . . . . . . . . . . . . . . . . . . . . . . . . . . . . . . . . . 1

ABSTRACT . . . . . . . . . . . . . . . . . . . . . . . . . . . . . . . . . . . . . . 1

BACKGROUND . . . . . . . . . . . . . . . . . . . . . . . . . . . . . . . . . . . .

OBJECTIVES . . . . . . . . . . . . . . . . . . . . . . . . . . . . . . . . . . . . . . .

METHODS . . . . . . . . . . . . . . . . . . . . . . . . . . . . . . . . . . . . . .

ACKNOWLEDGEMENTS . . . . . . . . . . . . . . . . . . . . . . . . . . . . . . . . . . . . . . .

REFERENCES . . . . . . . . . . . . . . . . . . . . . . . . . . . . . . . . . . . . . . 7

APPENDICES . . . . . . . . . . . . . . . . . . . . . . . . . . . . . . . . . . . . . 8

CONTRIBUTIONS OF AUTHORS . . . . . . . . . . . . . . . . . . . . . . . . . . . . . . . . . . . . . . . 9.9

DECLARATIONS OF INTEREST . . . . . . . . . . . . . . . . . . . . . . . . . . . . . . . . 10

SOURCES OF SUPPORT . . . . . . . . . . . . . . . . . . . . . . . . . . . . . . . . . . . . . . .

NOTES . . . . . . . . . . . . . . . . . . . . . . . . . . . . . . . . . . . . . . . . 10

Transperitoneal versus retroperitoneal laparoscopic adrenalectomy for adrenal tumours in adults (Protocol)

Copyright $\odot 2015$ The Cochrane Collaboration. Published by John Wiley \& Sons, Ltd. 


\title{
[Intervention Protocol] \\ Transperitoneal versus retroperitoneal laparoscopic adrenalectomy for adrenal tumours in adults
}

\author{
Alberto Arezzo $^{1}$, Giovanni G Cochetti ${ }^{2}$, Roberto Cirocchi ${ }^{3}$, Justus J Randolph ${ }^{4}$, Ettore E Mearini ${ }^{2}$, Roberto Passera ${ }^{5}$ \\ ${ }^{1}$ Department of Surgical Sciences, University of Torino, Turin, Italy. ${ }^{2}$ Surgical and Biomedical Science, University of Perugia, Perugia, \\ Italy. ${ }^{3}$ Department of General Surgery, University of Perugia, Terni, Italy. ${ }^{4}$ Tift College of Education, Mercer University, Atlanta, GA, \\ USA. ${ }^{5}$ Division of Nuclear Medicine, University of Torino, Torino, Italy \\ Contact address: Alberto Arezzo, Department of Surgical Sciences, University of Torino, Corso Achille Mario Dogliotti 14, Turin, \\ 10126, Italy. alberto.arezzo@unito.it. alberto.arezzo@mac.com.
}

Editorial group: Cochrane Metabolic and Endocrine Disorders Group.

Publication status and date: New, published in Issue 4, 2015.

Citation: Arezzo A, Cochetti GG, Cirocchi R, Randolph JJ, Mearini EE, Passera R. Transperitoneal versus retroperitoneal laparoscopic adrenalectomy for adrenal tumours in adults. Cochrane Database of Systematic Reviews 2015, Issue 4. Art. No.: CD011668. DOI: 10.1002/14651858.CD011668.

Copyright (C) 2015 The Cochrane Collaboration. Published by John Wiley \& Sons, Ltd.

\begin{abstract}
A B S T R A C T
This is the protocol for a review and there is no abstract. The objectives are as follows:

To assess the effects of laparoscopic transperitoneal adrenalectomy versus laparoscopic retroperitoneal adrenalectomy for adrenal tumours in adults.
\end{abstract}

\section{B A C K G R O U N D}

\section{Description of the condition}

Tumours or masses of the adrenal gland are quite common and usually unilateral. They may originate from the adrenal corticoid or the adrenal medulla of the gland. They are categorised as either functional (hormone-secreting) or silent (non hormone-secreting). Moreover, as any tumoural tissue, they may be benign or malignant. The majority of adrenocortical tumours are benign, non-functioning adenomas that are discovered incidentally on abdominal imaging studies. This is usually called an adrenal 'incidentaloma'. Hormone-secreting adrenocortical tumours are generally discovered by their symptoms causing Cushing's syndrome (showing enhanced secretion of adrenocorticotropic hormone, primary aldosteronism, or much less commonly, virilization. Carcinomas of the adrenal corticoid are quite rare, but sometimes aggressive. They may also be functional and cause Cushing's syndrome, virilization or both, or be nonfunctional and present as an abdominal mass or an incidental finding. More rarely tumours arise from the medulla of the gland. These are called phaeochromocytomas. Phaeochromocytomas are catecholamine-secreting tumours that arise from chromaffin cells of the adrenal medulla. They may be benign or malignant.

\section{Description of the intervention}

Adrenalectomy is usually recommended if the adrenal mass is four $\mathrm{cm}$ or greater in diameter, if the mass enlarges by one $\mathrm{cm}$ or more during the period of observation, or if evidence of autonomous hormonal secretion develops (Young 2007). Open transperitoneal adrenalectomy has been the gold standard of treatment for adrenal 
disease until 1992, when laparoscopic adrenalectomy was first described (Gagner 1992). After induction of anaesthesia, the patient is placed in lateral decubitus position with the affected side elevated around $60^{\circ}$, usually with the help of a bean bag. The ipsilateral arm is generally supported by a metal L-shaped support that is secured to the table.

Laparoscopic transperitoneal adrenalectomy (was first described in 1992 (Gagner 1992). The transperitoneal technique is performed with three to five transperitoneal ports through the anterior abdominal wall. After exposition of the adrenal gland space, the retroperitoneum is incised and the gland dissected. On the left side the descending colon is reflected medially, the adrenal vein is divided with bipolar diathermy or cutting between clips and dissection is carried out starting just superior to the renal vein until the adrenal gland is free. On the right side liver retraction is mandatory, while the colon rarely requires mobilisation. Here, the peritoneum is incised along the lateral aspect of the inferior vena cava, down to the superior edge of the renal vein. The short right adrenal vein is identified and divided between clips. When then dissection of the gland is completed at the lateral side the specimen is removed within a bag.

Laparoscopic retroperitoneal adrenalectomy was introduced in 1995 (Mercan 1995). This technique consists of approaching the adrenal gland directly through the retroperitoneal space, while not breaching the peritoneum. Patients are placed in lateral decubitus position and the table flexed in order to expand the operating space between the $12^{\text {th }}$ rib and the iliac crest. To obtain a retroperitoneal access a one $\mathrm{cm}$ skin incision is made below the 12th rib and a potential space is created below the fascia by careful finger dissection. On the left side the Gerota's fascia is incised around the superior aspect of the kidney and dissection is continued medially along the renal vein, until the main adrenal vein is encountered and divided between clips until mobilisation is completed. On the right side, too, the investing fascial layer is opened transversely along the upper renal pole until the inferior vena cava is identified to continue dissection superiorly along its lateral edge. Here, the right adrenal vein is encountered and divided between clips and the mobilisation of the gland is completed.

\section{Adverse effects of the intervention}

Intraoperative bleeding may occur in $1 \%$ to $4 \%$ of cases, depending on the different techniques used (Constantinides 2012). Small liver injuries can occur during retraction or adrenal dissection on the right, which might be controlled by bipolar coagulation, simple compression, or placement of fibrin material to achieve haemostasis, but sometimes, although rarely, may lead to conversion to open surgery. Vascular injuries, especially to the inferior vena cava, comprise almost $7 \%$ of all complications and are the leading cause for conversion (Corcione 2001). Small injuries may be compressed and coagulant agents applied. Increasing the intraabdominal pressure helps to control bleeding. If bleeding contin- ues, laparoscopic suturing should be attempted only if the surgeon has sufficient experience and skills, otherwise conversion to open surgery is mandatory. Pleural injuries and pneumothoraces may occur occasionally, requiring direct diaphragm suture closure (Naito 1995). A chest tube placement is rarely required initially, although it may be required later if a significant pneumothorax develops. Injury to the spleen and the left side of the pancreas are also reported (Greco 2011; Terachi 2000).

\section{How the intervention might work}

Numerous studies have shown the safety and feasibility of the laparoscopic technique since its introduction in 1992. Several benefits were shown compared to open procedures: a decreased hospital stay, faster recovery, decreased pain and narcotic use, and fewer complications (Assalia 2004). Minimally invasive adrenalectomy is now considered the standard treatment for benign adrenal masses (Jacobs 1997). Laparoscopic retroperitoneal adrenalectomy was first performed and described in 1995 (Mercan 1995). By directly entering the retroperitoneal space, and not breaching the peritoneum, this technique was advocated by the authors potentially resulting in a shorter operative time, less blood loss, less postoperative pain and shorter hospital stay (Constantinides 2012). Despite favourable results reported for both minimally invasive adrenalectomy techniques using either the transperitoneal or retroperitoneal route, only a few studies have compared the two procedures thoroughly, showing no superiority of either technique. However, most studies have been limited by a small sample size and a single-institution design.

\section{Why it is important to do this review}

The intrinsic difficulties of laparoscopic transperitoneal adrenalectomy limited the diffusion of the technique which was performed in less than $20 \%$ of cases until 2006 (Murphy 2010). In the following years, the demonstration of the general advantages of laparoscopy, such as reduced blood loss, shorter hospital stay, and faster return to normal activity (Guazzoni 1995), together with the accumulated experience inverted the trend in favour of the laparoscopic technique, so that today laparoscopic transperitoneal adrenalectomy has become the most widely utilised procedure for people with benign adrenal disease. Introduced in 1995, laparoscopic retroperitoneal adrenalectomy was proposed as a good alternative in selected cases and it was utilised more and more frequently (Mercan 1995). Potential advantages could be a shorter operative time, less postoperative pain and shorter hospital stay (Constantinides 2012). Several studies have compared the outcomes of laparoscopic transperitoneal adrenalectomy and laparoscopic retroperitoneal adrenalectomy, but their results are inconclusive because of their design and inclusion criteria. In addition, several meta-analyses have compared the transperitoneal with the 
retroperitoneal adrenalectomy (Chen 2013; Constantinides 2012; Nigri 2013). These analyses, however, were not fully reliable, because the number of the included studies and the study population were rather small and heterogeneous. Moreover, they presented a mixture of observational and interventional studies. Furthermore, recent randomised trials could not be included as the last search conducted was anterior to their publication (Barczyn ski 2014; Mohammadi-Fallah 2013). Finally, no sound risk of bias evaluation was performed. Therefore, our systematic review will try to establish a reliable body of evidence of relevant outcomes in people undergoing laparoscopic transperitoneal adrenalectomy or laparoscopic retroperitoneal adrenalectomy.

\section{O B J E C T I VES}

To assess the effects of laparoscopic transperitoneal adrenalectomy versus laparoscopic retroperitoneal adrenalectomy for adrenal tumours in adults.

\section{METHODS}

\section{Criteria for considering studies for this review}

\section{Types of studies}

We will include randomised controlled clinical trials (RCTs).

\section{Types of participants}

We will include data from adult (older than 18 years) individuals who underwent laparoscopic transperitoneal adrenalectomy or laparoscopic retroperitoneal adrenalectomy for preoperatively assessed adrenal tumours.

\section{Diagnostic criteria}

Corticoid and medullary, benign and malignant, functional and silent tumours or masses of the adrenal gland assessed by both laboratory and imaging studies.

\section{Types of interventions}

We plan to investigate the following comparisons of intervention versus control/comparator.

\section{- Intervention}

- Laparoscopic retroperitoneal adrenalectomy defined as any technique approaching the adrenal gland directly through the retroperitoneal space, and not breaching the peritoneum.
- Comparator

- Laparoscopic transperitoneal adrenalectomy defined as any technique approaching the adrenal gland directly through the abdominal wall and peritoneal sac.

Concomitant interventions will have to be the same in the intervention and comparator groups to establish fair comparisons.

\section{Types of outcome measures}

\section{Primary outcomes}

- All-cause mortality.

- All-cause morbidity.

- Adverse events.

\section{Secondary outcomes}

- Operative parameters

- Duration of surgery.

- Operative blood loss.

- Intraoperative bleeding.

- Conversion to open surgery.

- Postoperative parameters

- Time to oral fluid or food intake.

- Time to full ambulation.

- Length of hospital stay.

- Pneumothorax/haemothorax.

- Chest infection/pleural effusion.

- Splenic injury.

- Abdominal abscess.

- Health-related quality of life.

- Socioeconomic effects.

\section{Method and timing of outcome measurement}

- All-cause mortality: defined as death from any cause and measured within 30 days after surgery and thereafter.

- All-cause morbidity: defined as any deviation from the regular post-operative course (e.g. chest infection or splenic injury) and measured within 30 days after surgery and thereafter.

- Adverse events: any undesirable experience happening during the trial measured within 30 days after surgery and thereafter..

- Duration of surgery: defined as the duration of general anaesthesia and measured at the end of general anaesthesia.

- Operative blood loss: defined as the maximum decrease in haemoglobin and measured at the end of surgery and thereafter.

- Intraoperative bleeding: defined as the occurrence of blood loss greater than $200 \mathrm{ml}$ and measured at the end of surgery.

- Conversion to open surgery: defined as any technical failure requiring a larger skin incision before the complete dissection of the gland and measured at the end of surgery. 
- Time to oral fluid or food intake: defined as the time to oral intake of fluids or food and measured within one week after surgery and thereafter.

- Lenght of hospital stay: defined as the time to discharge from hospital and measured within 30 days after surgery and thereafter.

- Pneumothorax/haemothorax: defined as any detection of a collection of air, gas or blood in the chest or pleural space that causes part or all of a lung to collapse and measured within 30 days after surgery and thereafter.

- Chest infection/pleural effusion: defined as any diagnosis of infection that affects lungs (either larger or smaller air sacs) or the accumulation of fluid in the pleural space and measured within 30 days after surgery and thereafter.

- Splenic injury: defined as any splenic trauma determining splenic capsule tear or parenchymal hematoma and measured at the end of surgery and thereafter.

- Health-related quality of life: evaluated by a validated instrument such as the Short-Form Survey (SF-36) and measured within the first week, at 30 after surgery and thereafter.

- Socioeconomic effects: such as length of hospital stay, time to return to normal activity and time to return to work and measured within 30 days after surgery and thereafter.

\section{'Summary of findings' table}

We will present a 'Summary of findings table' reporting the following outcomes listed according to priority.

1. All-cause mortality.

2. All-cause morbidity.

3. Adverse events (including some operative or postoperative parameters).

4. Health-related quality of life.

5. Socioeconomic effects.

\section{Search methods for identification of studies}

\section{Electronic searches}

We will search the following sources from inception of each database to the specified date and will place no restrictions on the language of publication.

- Cochrane Library.

- Cochrane Database of Systematic Reviews (CDSR)

(CENTRAL)

- Cochrane Central Register of Controlled Trials

- Database of Abstracts of Reviews of Effects (DARE)

- Health Technology Assessment (HTA)

- MEDLINE

- EMBASE.

- ClinicalTrials.gov (https://clinicaltrials.gov/).
- World Health Organization (WHO) International Clinical Trials Registry Platform (ICTRP) Search Protal (http:// apps.who.int/trialsearch/), which is a meta-register of studies including several trial registers:

- Australian New Zealand Clinical Trials Registry

- ClinicalTrials.gov

- European Union (EU) Clinical Trials Register

- International Standard Randomised Controlled Trial

Number (ISRCTN) registry

- Brazilian Clinical Trials Registry

- Chinese Clinical Trial Registry

- Clinical Trials Registry - India

- Clinical Research Information Service - Republic of

Korea
- Cuban Public Registry of Clinical Trials
- German Clinical Trials Register
- Iranian Registry of Clinical Trials
- Japan Primary Registries Network
- Pan African Clinical Trial Registry
- Sri Lanka Clinical Trials Registry
- The Netherlands National Trial Register
- Thai Clinical Trials Register

We will continuously apply a MEDLINE (via Ovid SP) email alert service established by the Cochrane Metabolic and Endocrine Disorders (CMED) Group to identify newly published studies using the same search strategy as described for MEDLINE (for details on search strategies see Appendix 1). After supplying the final review draft for editorial approval, the CMED Group will perform a complete updated search on all databases available at the editorial office and send the results to the review authors. Should we identify new studies for inclusion, we will evaluate these, incorporate the findings into our review, and resubmit another review draft (Beller 2013).

If we detect additional relevant key words during any of the electronic or other searches, we will modify the electronic search strategies to incorporate these terms and document the changes.

\section{Searching other resources}

We will attempt to identify other potentially-eligible trials or ancillary publications by searching the reference lists of retrieved included trials, (systematic) reviews, meta-analyses and health technology assessment reports. We will also contact study authors of included trials in order to identify any further studies that we may have missed.

\section{Data collection and analysis}

\section{Selection of studies}


Two review authors (AA, RC) will independently scan the abstract, title, or both, of every record retrieved, to determine which studies should be assessed further. We will investigate all potentially-relevant articles as full text. We will resolve any discrepancies through consensus or recourse to a third review author (RP). If we cannot resolve a disagreement, we will add the study as a 'study awaiting classification' and we will contact study authors for clarification. We will present an adapted Preferred Reporting Items for Systematic Reviews and Meta-Analyses (PRISMA) flow diagram showing the process of study selection (Liberati 2009).

\section{Data extraction and management}

For studies that fulfil inclusion criteria, two review authors (AA, $\mathrm{RC}$ ) will independently extract key participant and intervention characteristics and report data on efficacy outcomes and adverse events using standard data extraction templates as supplied by the CMED Group, with any disagreements to be resolved by discussion, or, if required, by consultation with a third review author (RP).

We will provide information including trial identifier about potentially relevant ongoing studies in the 'Characteristics of ongoing studies' table and in a joint appendix. We will try to find the protocol for each included study and will report primary, secondary, and other outcomes in comparison with data in publications in a joint appendix 'Matrix of study endpoint (publications and trial documents)'.

We will email all authors of included studies to enquire whether they are willing to answer questions regarding their trials. We will present the results of this survey in an appendix. We will thereafter seek relevant missing information on the trial from the primary author(s) of the article, if required.

\section{Dealing with duplicate and companion publications}

In the event of duplicate publications, companion documents or multiple reports of a primary study, we will maximise yield of information by collating all available data and use the most complete dataset aggregated across all known publications.

\section{Assessment of risk of bias in included studies}

Two review authors (AA, RC) will independently assess the risk of bias of each included study. We will resolve any disagreements by consensus, or by consultation with a third review author (RP).

We will use the Cochrane Collaboration's tool for assessing risk of bias (Higgins 2011a; Higgins 2011b), and will evaluate the following criteria.

- Random sequence generation (selection bias).

- Allocation concealment (selection bias).

- Imbalances in baseline characteristics (chance bias).

- Blinding of participants and personnel (performance bias).

- Blinding of outcome assessment (detection bias).
- Incomplete outcome data (attrition bias).

- Selective reporting (reporting bias).

- Other potential sources of bias.

We will judge the above 'Risk of bias' criteria as 'low risk', 'high risk' or 'unclear risk' and will evaluate individual bias items as described in the Cochrane Handbook for Systematic Reviews of Interventions (Higgins 2011a). We will present a risk of bias graph and a risk of bias summary. We will assess the impact of individual bias domains on study results at the endpoint and study levels. In case of high risk of selection bias, we will mark all endpoints investigated in the associated study as high risk.

We will evaluate whether imbalances in baseline characteristics existed and how these were addressed (Egbewale 2014; Riley 2013). For performance bias (blinding of participants and personnel) and detection bias (blinding of outcome assessors) we will evaluate the risk of bias separately for each outcome (Hróbjartsson 2013). We will note whether endpoints were self-reported, investigatorassessed, or adjudicated outcome measures.

We will consider the implications of missing outcome data from individual participants per outcome such as high drop-out rates (e.g. above $15 \%$ ) or disparate attrition rates (e.g. difference of $10 \%$ or more between study arms).

We will assess outcome reporting bias by integrating the results of the appendix 'Matrix of study endpoints (publications and trial documents)' (Boutron 2014; Mathieu 2009), and the appendix 'Examination of outcome reporting bias' (Kirkham 2010). This analysis will form the basis of the judgement of selective reporting (reporting bias).

We will distinguish between self-reported, investigator-assessed and adjudicated outcome measures.

We define the following endpoints as self-reported outcomes.

- Adverse events, measured by participants.

- Health-related quality of life.

We define the following endpoints as investigator-assessed outcomes.

- All-cause mortality

- All-cause morbidity

- Adverse events, measured by study personnel.

- Operative and postoperative parameters.

- Socioeconomic effects.

\section{Measures of treatment effect}

We will express dichotomous data as odds ratios (ORs) or risk ratios (RRs) with 95\% confidence intervals (CIs). We will express continuous data as mean differences (MDs) with $95 \%$ CIs. We will express time-to-event data as hazard ratios (HRs) wit $95 \%$ CIs.

\section{Unit of analysis issues}


We will take into account the level at which randomisation occurred, such as cross-over trials, cluster-randomised trials and multiple observations for the same outcome.

\section{Dealing with missing data}

If possible, we will obtain missing data from study authors, and carefully evaluate important numerical data such as screened, randomised participants as well as intention-to-treat (ITT), and astreated and per-protocol populations. We will investigate attrition rates, e.g. drop-outs, losses to follow up and withdrawals, and critically appraise issues of missing data and imputation methods (e.g. last observation carried forward (LOCF)).

Where means and standard deviations for outcomes have not been reported and we have not received the needed information from study authors, we will impute these values by estimating the mean and variance from the median, range, and the size of the sample (Hozo 2005), or by assuming the standard deviation of the missing outcome to be the average of the standard deviations from those studies where this information was reported.

We will investigate the impact of imputation on meta-analyses by means of sensitivity analysis.

\section{Assessment of heterogeneity}

In the event of substantial clinical, methodological or statistical heterogeneity, we will not report study results as the pooled effect estimate in a meta-analysis.

We will identify heterogeneity (inconsistency) through visual inspection of the forest plots and by using a standard $\mathrm{Chi}^{2}$ test with a significance level of $\alpha=0.1$. In view of the low power of this test, we will also consider the $\mathrm{I}^{2}$ statistic, which quantifies inconsistency across studies to assess the impact of heterogeneity on the meta-analysis (Higgins 2002; Higgins 2003); where an I² statistic of $75 \%$ or more indicates a considerable level of heterogeneity (Higgins 2011a).

When we find heterogeneity, we will attempt to determine possible reasons for it by examining individual study and subgroup characteristics.

\section{Assessment of reporting biases}

If we include 10 or more studies investigating a particular outcome, we will use funnel plots to assess small-study effects. There can be several explanations for funnel plot asymmetry, including true heterogeneity of effect with respect to trial size, poor methodological design (and hence bias of small trials), and publication bias. We will therefore interpret results carefully (Sterne 2011).

\section{Data synthesis}

Unless there is good evidence for homogeneous effects across studies, we will summarise primarily low risk of bias data using a random-effects model (Wood 2008). We will interpret random-effects meta-analyses with due consideration of the whole distribution of effects, ideally by presenting a prediction interval (Higgins 2009). A prediction interval specifies a predicted range for the true treatment effect in an individual study (Riley 2011). We will also perform statistical analyses according to the statistical guidelines contained in the Cochrane Handbook for Systematic Reviews of Interventions (Higgins 2011a).

\section{Quality of evidence}

We will present the overall quality of the evidence for each outcome according to the Grading of Recommendations Assessment, Development and Evaluation (GRADE) approach which takes into account issues not only related to internal validity (risk of bias, inconsistency, imprecision, publication bias) but also to external validity such as directness of results. Two review authors (AA, RC) will independently rate the quality for each outcome. We will present a summary of the evidence in a 'Summary of findings' table, which provides key information about the best estimate of the magnitude of the effect, in relative terms and absolute differences, for each relevant comparison of alternative management strategies, numbers of participants, and studies addressing each important outcome and the rating of the overall confidence in effect estimates for each outcome. We will create the 'Summary of findings' table based on the methods described in the Cochrane Handbook for Systematic Reviews of Interventions (Higgins 2011a). We will present results for the outcomes as described in Types of outcome measures. If meta-analysis is not possible, we will present results in a narrative 'Summary of findings' table .

We will also establish a 'Checklist to aid consistency and reproducibility of GRADE assessments' to help with standardisation of 'Summary of findings' tables (Meader 2014).

\section{Subgroup analysis and investigation of heterogeneity}

We expect the following characteristics to introduce clinical heterogeneity and plan to carry out subgroup analyses with investigation of interactions.

- Tumour size: $<6 \mathrm{~cm}$ versus $>6 \mathrm{~cm}$.

- Previous abdominal surgery: yes/no.

- Body mass index (BMI): $<30 \mathrm{~kg} / \mathrm{m}^{2}$ versus $\geq 30 \mathrm{~kg} / \mathrm{m}^{2}$.

\section{Sensitivity analysis}

We plan to perform sensitivity analyses in order to explore the influence of the following factors (when applicable) on effect sizes by restricting the analysis to:

- Published studies.

- Taking into account risk of bias, as specified in the 'Assessment of risk of bias in included studies' section. 
- Very long or large studies to establish the extent to which they dominate the results.

- Studies using the following filters: diagnostic criteria, imputation, language of publication, source of funding (industry versus other), or country.

We will also test the robustness of the results by repeating the analysis using different measures of effect size (RRs, ORs etc.) and different statistical models (fixed-effect and random-effects models).

\section{ACKNOW LEDGEMENTS}

We would like to thank Lorenzo Causarano for helping us in the search strategy providing the correct syntax.

\section{R E F E R E N C E S}

\section{Additional references}

\section{Assalia 2004}

Assalia A, Gagner M. Laparoscopic adrenalectomy. British Journal of Surgery 2004;91:1259-74.

\section{Barczyń ski 2014}

Barczyn ski M, Konturek A, Nowak W. Randomized clinical trial of posterior retroperitoneoscopic adrenalectomy versus lateral transperitoneal laparoscopic adrenalectomy with a 5year follow-up. Annals of Surgery 2014;260(5):740-7.

\section{Beller 2013}

Beller EM, Chen JK, Wang UL, Glasziou PP. Are systematic reviews up-to-date at the time of publication?. Systematic Reviews 2013;2(1):36.

\section{Boutron 2014}

Boutron I, Altman DG, Hopewell S, Vera-Badillo F, Tannock I, Ravaud P. Impact of spin in the abstracts of articles reporting results of randomized controlled trials in the field of cancer: the SPIIN randomized controlled trial. Journal of Clinical Oncology 2014;32:4120-6.

\section{Chen 2013}

Chen W, Li F, Chen D. Retroperitoneal versus transperitoneal laparoscopic adrenalectomy in adrenal tumour: a meta-analysis. Surgical Laparoscopy, Endoscopy \& Percutaneous Techniques 2013;23:121-7.

\section{Constantinides 2012}

Constantinides VA, Christakis I, Touska P, Palazzo FF. Systematic review and meta-analysis of retroperitoneoscopic versus laparoscopic adrenalectomy. British Journal of Surgery 2012;99:1639-48.

\section{Corcione 2001}

Corcione F, Esposito C, Cuccurullo D, Settembre A, Fusco F, Bianco A. Vena cava injury: A serious complication during right adrenalectomy. Surgical Endoscopy 2001;15: 218.

\section{Egbewale 2014}

Egbewale BE, Lewis M, Sim J. Bias, precision and statistical power of analysis of covariance in the analysis of randomized trials with baseline imbalance: a simulation study. $B M C$ Medical Research Methodology 2014;14:49:49. [DOI: 10.1186/1471-2288-14-49]

\section{Gagner 1992}

Gagner M, Lacroix A, Bolté E. Laparoscopic adrenalectomy in Cushing's syndrome and pheochromocytoma. New England Journal of Medicine 1992;327(14):1033.

\section{Greco 2011}

Greco F, Hoda MR, Rassweiler J, Fahlenkamp D, Neisius DA, Kutta A. Laparoscopic adrenalectomy in urological centres - the experience of the German Laparoscopic Working Group. BJU International 2011;108:1646-51.

\section{Guazzoni 1995}

Guazzoni G, Montorsi F, Bocciardi A. Transperitoneal laparoscopic versus open adrenalectomy for benign hyperfunctioning adrenal tumours: a comparative study. Journal of Urology 1995;153:1597-600.

\section{Higgins 2002}

Higgins JPT, Thompson SG. Quantifying heterogeneity in a meta-analysis. Statistics in Medicine 2002;21:1539-58.

\section{Higgins 2003}

Higgins JPT, Thompson SG, Deeks JJ, Altman DG. Measuring inconsistency in meta-analysis. BMJ 2003;327: 557-60.

\section{Higgins 2009}

Higgins JPT, Thompson SG, Spiegelhalter DJ. A reevaluation of random-effects meta-analysis. Journal of The Royal Statistical Society. Series A 2009;172(1):137-59.

\section{Higgins 2011a}

Higgins JPT, Green S (editors). Cochrane Handbook for Systematic Reviews of Interventions Version 5.1.0 [updated March 2011]. The Cochrane Collaboration, 2011. Available from www.cochrane-handbook.org.

\section{Higgins 2011b}

Higgins JPT, Altman DG, Gøtzsche PC, Jüni P, Moher D, Oxman AD, et al. The Cochrane Collaboration's tool for assessing risk of bias in randomised trials. BMJ 2011;343: d5928.

\section{Hozo 2005}

Hozo SP, Djulbegovic B, Hozo I. Estimating the mean and variance from the median, range, and the size of a sample. BMC Medical Research Methodology 2005;5:13. 


\section{Hróbjartsson 2013}

Hróbjartsson A, Thomsen AS, Emanuelsson F, Tendal B, Hilden J, Boutron I, et al. Observer bias in randomized clinical trials with measurement scale outcomes: a systematic review of trials with both blinded and nonblinded assessors. Canadian Medical Association Journal 2013;185 (4):E201-11.

\section{Jacobs 1997}

Jacobs JK, Goldstein RE, Geer RJ. Laparoscopic adrenalectomy: a new standard of care. Annals of Surgery 1997;225:495-501.

\section{Kirkham 2010}

Kirkham JJ, Dwan KM, Altman DG, Gamble C, Dodd $S$, Smyth R, et al. The impact of outcome reporting bias in randomised controlled trials on a cohort of systematic reviews. BMJ 2010;340:c365. [DOI: 10.1136/bmj.c365]

\section{Liberati 2009}

Liberati A, Altman DG, Tetzlaff J, Mulrow C, Gøtzsche PC, Ioannidis JPA, et al. The PRISMA statement for reporting systematic and meta-analyses of studies that evaluate interventions: explanation and elaboration. PLoS Medicine 2009;6(7):1-28. [DOI: 10.1371/journal.pmed.1000100]

\section{Mathieu 2009}

Mathieu S, Boutron I, Moher D, Altman DG, Ravaud P. Comparison of registered and published primary outcomes in randomized controlled trials. JAMA 2009;302:977-84.

\section{Meader 2014}

Meader N, King K, Llewellyn A, Norman G, Brown J, Rodgers $\mathrm{M}$, et al. A checklist designed to aid consistency and reproducibility of GRADE assessments: development and pilot validation. Systematic Reviews 2014;3:82.

\section{Mercan 1995}

Mercan S, Seven R, Ozarmagan S, Tezelman S. Endoscopic retroperitoneal adrenalectomy. Surgery 1995;118:1071-6.

\section{Mohammadi-Fallah 2013}

Mohammadi-Fallah MR, Mehdizadeh A, Badalzadeh A, Izadseresht B, Dadkhah N, Barbod A, et al. Comparison of transperitoneal versus retroperitoneal laparoscopic adrenalectomy in a prospective randomized study. Journal of Laparoendoscopic \& Advanced Surgical Techniques 2013; 23(4):362-6.
Murphy 2010

Murphy MM, Witkowski ER, Ng SC. Trends in adrenalectomy: a recent national review. Surgical Endoscopy and Other Interventional Techniques 2010;24:2518-26.

\section{Naito 1995}

Naito S, Uozumi J, Shimura H, Ichimiya H, Tanaka M, Kumazawa J. Laparoscopic adrenalectomy: review of 14 cases and comparison with open adrenalectomy. Journal of Endourology 1995;9:491-5.

\section{Nigri 2013}

Nigri G, Rosman AS, Petrucciani N. Meta-analysis of trials comparing laparoscopic transperitoneal and retroperitoneal adrenalectomy. Surgery 2013;153:111-9.

\section{Riley 2011}

Riley RD, Higgins JP, Deeks JJ. Interpretation of random effects meta-analyses. BMJ 2011;342:d549.

\section{Riley 2013}

Riley RD, Kauser I, Bland M, Thijs L, Staessen JA, Wang J et al. Meta-analysis of randomised trials with a continuous outcome according to baseline imbalance and availability of individual participant data. Statistics in Medicine 2013;32: 2747-66.

\section{Sterne 2011}

Sterne JA, Sutton AJ, Ioannidis JP, Terrin N, Jones DR, Lau $\mathrm{J}$, et al. Recommendations for examining and interpreting funnel plot asymmetry in meta-analyses of randomised controlled trials. BMJ 2011;343:d4002.

\section{Terachi 2000}

Terachi T, Yoshida O, Matsuda T, Orikasa S, Chiba Y, Takahashi K. Complications of laparoscopic and retroperitoneoscopic adrenalectomies in 370 cases in Japan: a multi-institutional study. Biomedicine \& Pharmacotherapy 2000;54(Suppl 1):211s-214s.

\section{Wood 2008}

Wood L, Egger M, Gluud LL, Schulz KF, Juni P, Altman DG, et al. Empirical evidence of bias in treatment effect estimates in controlled trials with different interventions and outcomes: meta-epidemiological study. BMJ 2008;336 (7644):601-5.

Young 2007

Young WF Jr. The incidentally discovered adrenal mass. New England Journal of Medicine 2007;356:601-10.

* Indicates the major publication for the study 
A P P E N D I CES

\section{Appendix I. Search strategies}

\section{The Cochrane Library}

\#1 ((transperiton* or retroperiton* or laparoscop* or endoscop $\left.{ }^{*}\right)$ near/7 adrenalectom*):ti,ab,kw

\#2 [mh "Adrenalectomy"] and [mh "Laparoscopy"]

\#3 \#1 or \#2

\section{MEDLINE (Ovid SP)}

1 ((transperiton* or retroperiton* or laparoscop* or endoscop*) adj6 adrenalectom*).tw.

2 Adrenalectomy/ and exp Laparoscopy/

31 or 2

4 randomized controlled trial.pt.

5 controlled clinical trial.pt.

6 randomi?ed.ab.

7 placebo.ab.

8 randomly.ab.

9 trial.ab.

10 groups.ab.

11 or/4-10

12 exp animals/ not humans/

1311 not 12

143 and 13

\section{EMBASE (Ovid SP)}

1. ((transperiton* or retroperiton* or laparoscop* or endoscop*) adj6 adrenalectom*).tw.

2. random*..tw. or clinical trial*.mp. or exp health care quality/

3. 1 and 2

4. limit 3 to embase

\section{ClinicalTrials.gov (Basic Search)}

(transperitoneal OR retroperitoneal OR laparoscopic OR endoscopic OR retroperitoneoscopic) AND (adrenalectomy OR adrenalectomies)

\section{ICTRP Search Portal (Standard Search)}

transperiton* AND adrenalectom* OR retroperiton* AND adrenalectom* OR laparoscop* AND adrenalectom* OR endoscop* AND adrenalectom* 


\section{CONTRIBUTIONSOFAUTHORS}

Alberto Arezzo (AA): protocol drafting, search strategy development.

Giovanni G Cochetti (GC): protocol drafting.

Roberto Cirocchi (RC): protocol drafting.

Justus J Randolph (JR): protocol drafting.

Ettore E Mearini (EM): protocol drafting.

Roberto Passera (RP): protocol drafting.

\section{DECLARATIONSOF INTEREST}

AA: none known.

GC: none known.

RC: none known.

JR: none known.

EM: none known.

RP: none known.

\section{SOURCES OF SUPPORT}

\section{Internal sources}

- none, Other.

\section{External sources}

- No sources of support supplied

\section{NOT E S}

We have based parts of the Methods and Appendix 1 sections of this Cochrane protocol on a standard template established by the CMED Group. 